\title{
Soluble Thy-1 reverses lung fibrosis via its integrin-binding motif
}

\author{
Chunting Tan, ${ }^{1,2}$ Min Jiang, ${ }^{3}$ Simon S. Wong, ${ }^{1}$ Celia R. Espinoza, ${ }^{1}$ Ceonne Kim, ${ }^{1}$ Xiaoping Li, ${ }^{1}$ \\ Edward Connors, ${ }^{1}$ and James S. Hagood ${ }^{1,4}$ \\ 'Department of Pediatrics, Division of Respiratory Medicine, UCSD, San Diego, California, USA. ${ }^{2}$ Department of Respiratory \\ Medicine, Beijing Friendship Hospital, Capital Medical University, Beijing, China. ${ }^{3}$ Department of Pediatrics, First Affiliated \\ Hospital of Guangxi Medical University, Nanning, Guangxi, China. ${ }^{4}$ Department of Pediatrics, University of North Carolina \\ at Chapel Hill, Chapel Hill, North Carolina, USA.
}

Loss of Thy-1 expression in fibroblasts correlates with lung fibrogenesis; however, the clinical relevance of therapeutic targeting of myofibroblasts via Thy-1-associated pathways remains to be explored. Using single (self-resolving) or repetitive (nonresolving) intratracheal administration of bleomycin in type 1 collagen-GFP reporter mice, we report that Thy- 1 surface expression, but not mRNA, is reversibly diminished in activated fibroblasts and myofibroblasts in self-resolving fibrosis. However, Thy-1 mRNA expression is silenced in lung with nonresolving fibrosis following repetitive bleomycin administration, associated with persistent activation of $\alpha \mathrm{v}$ integrin. Thy1-null mice showed progressive $\alpha v$ integrin activation and myofibroblast accumulation after a single dose of bleomycin. In vitro, targeting of $\alpha \mathrm{v}$ integrin by soluble Thy-1-Fc (sThy-1), but not RLEmutated Thy- 1 or IgG, reversed TCF- $\beta 1$-induced myofibroblast differentiation in a dose-dependent manner, suggesting that Thy-1's integrin-binding RCD motif is required for the reversibility of myofibroblast differentiation. In vivo, treatment of established fibrosis induced either by singledose bleomycin in WT mice or by induction of active TCF- $\beta 1$ by doxycycline in Cc10-rtTA-tTS-Tgfb1 mice with sThy-1 (1000 $\mathrm{ng} / \mathrm{kg}$, i.v.) promoted resolution of fibrosis. Collectively, these findings demonstrate that sThy- 1 therapeutically inhibits the $\alpha v$ integrin-driven feedback loop that amplifies and sustains fibrosis.

Authorship note: CT, M], and SSW contributed equally to this work and are co-first authors.

Conflict of interest: The authors have declared that no conflict of interest exists.

Copyright: (c) 2019, American Society for Clinical Investigation.

Submitted: June 24, 2019

Accepted: September 25, 2019

Published: November 1, 2019.

Reference information: JCI Insight. 2019;4(21):e131152.

https://doi.org/10.1172/jci.

insight.131152.

\section{Introduction}

Progressive pulmonary fibrosis occurs worldwide and is nearly uniformly fatal. Current FDA-approved medication has only decelerated the ongoing tissue remodeling, rather than halting or reversing it $(1,2)$. There are clearly significant gaps in understanding impaired tissue remodeling leading to progressive fibrosis, characterized by persistent or aberrant fibroblast activation and excessive deposition of extracellular matrix (ECM) in the lungs. It is clear that fibrotic tissue remodeling can progress via multiple feed-forward amplification loops if the effector cells, myofibroblasts, acquire certain fibrotic phenotype characteristics, such as apoptosis resistance $(3,4)$, restricted dedifferentiation $(5,6)$, and compromised autophagy (7). At the same time, excessive and persistent abnormal ECM promotes further activation of fibroblasts and differentiation to myofibroblasts (8). Therefore, interruption of ongoing fibroblast activation and elimination or inactivation of myofibroblasts are critical for fibrosis resolution.

Targeting of $\alpha \mathrm{v}$ integrin has identified a core molecular pathway that regulates organ fibrosis (9). $\alpha \mathrm{v}$ Integrins are expressed in collagen ${ }^{+}$fibroblasts and are upregulated and activated in $\alpha$ smooth muscle actin ${ }^{+}$ $\left(\alpha \mathrm{SMA}^{+}\right)$myofibroblasts, contractile cells capable of exerting force on tethered ligands, resulting in integrin-dependent activation of latent TGF- $\beta$ (10). Specifically, $\alpha$ v integrins interact with an arginine-glycine-aspartic acid (RGD) motif present in the latency-associated peptide, which maintains TGF- $\beta$ in an inactive state in the ECM. Active TGF- $\beta$ can be released from the latency-associated peptide following $\alpha \mathrm{v}$ integrin binding and myofibroblast contraction (11-13). In mice, genetic depletion of the $\alpha \mathrm{v}$ integrin subunit in myofibroblasts using the Pdgfrb-Cre system was protective against organ fibrosis, including lungs $(9,14)$. $\alpha \mathrm{v}$ Integrin blockade also reduces TGF- $\beta$ activation in primary human skeletal muscle and cardiac PDGFR $\beta^{+}$ cells (14). Clearly, these studies have indicated that $\alpha \mathrm{v}$ integrins are highly expressed and targetable on myofibroblasts, thus representing a potential novel therapeutic option in the treatment of fibrotic diseases. 
Thy-1, a GPI-anchored glycoprotein, contains an integrin-binding RGD-like motif (RLD) and regulates the phenotype of fibroblasts (15-18). Thy-1 is highly expressed on normal fibroblasts but is absent in activated fibroblasts and myofibroblasts in idiopathic pulmonary fibrosis (IPF) fibroblastic foci $(19,20)$ and decreases with aging (21). In vitro, Thy-1 expression determines the ability of lung fibroblasts to activate in response to fibrogenic stimuli (22). Thy-1- ${ }^{-}$lung fibroblasts are hyperproliferative (23), differentially express growth factor receptors (24), and display altered cytokine signaling (25), showing enhanced myofibroblastic differentiation (26), apoptosis resistance (27), and enhanced activation of latent TGF- $\beta$ (22, 28). Notably, interactions of soluble Thy-1 (sThy-1) with $\alpha v \beta 5$ integrin inhibited lung fibroblast contraction-induced latent TGF- $\beta 1$ activation and myofibroblast differentiation (28). Most recently, we found that Thy-1 physically couples to inactive $\alpha v \beta 3$ integrin, altering baseline integrin avidity to ECM ligands and facilitating preadhesion clustering of integrins and membrane rafts via Thy-1's glycophosphatidylinositol tether (29). Disruption of Thy-1- $\alpha \mathrm{v} \beta 3$ coupling altered recruitment of Src family kinases to adhesion complexes and impaired mechanosensitive, force-induced Rho signaling, and rigidity sensing, sufficiently to induce myofibroblast differentiation in normal, physiologically soft ECMs. These findings in vitro highlight the role of Thy- 1 in $\alpha$ v integrin-dependent TGF- $\beta 1$ activation and downstream profibrotic signaling in fibroblasts; however, its therapeutic relevance in pathological fibrosis has not been determined.

In this study, we show that Thy-1 expression in vivo was reversibly diminished in activated fibroblasts or myofibroblasts in self-resolving fibrosis. However, Thy-1 was silenced in myofibroblasts in progressive fibrosis, associated with persistent activation of $\alpha \mathrm{v}$ integrin, impaired tissue remodeling characterized by aberrant fibroblast activation, and excessive deposition of ECM. Moreover, targeting of av integrin by sThy-1, via an integrin-binding RLD, has therapeutic effectiveness in murine models of bleomycin- and TGF- $\beta 1$-induced fibrogenesis, indicative of therapeutic potential in reversing established lung fibrosis.

\section{Results}

Acute injury induced fibroblastic shedding of Thy-1 that did not interrupt lung fibrosis resolution. We initially examined Thy-1 expression in bleomycin-induced lung fibrosis in transgenic reporter Col-GFP mice expressing collagen- $\alpha 1(\mathrm{I})$ promoter/enhancer-driven GFP (Figure 1B). Lung fibrosis was induced by instilling bleomycin (intratracheally [i.t.], 4 unit/kg) or saline (control) and evaluated by measuring deposition of collagen and numbers of $\mathrm{GFP}^{+}$fibroblasts or $\mathrm{GFP}^{+} / \alpha \mathrm{SMA}^{+}$myofibroblasts. Bleomycin-treated mice developed severe fibrosis with the accumulation of $\mathrm{GFP}^{+}$cells at 28 days, which were significantly decreased following resolution of fibrosis at 56 days (Figure 1, A, C, and D). In this model, we found that Thy-1 immunostaining decreased mostly in $\mathrm{GFP}^{+}$fibroblasts and $\mathrm{GFP}^{+} / \alpha \mathrm{SMA}^{+}$myofibroblasts (data not shown) 28 days following bleomycin instillation (Figure 1, A and C). There were no significant changes of Thy-1 immunostaining in $\mathrm{GFP}^{-}$cells over time (data not shown). After 8 weeks, however, Thy-1 expression was recovered in scattered fibroblasts in alveolar septae, around blood vessels, and in some areas of pleura. Examination of mRNA expression by qPCR did not demonstrate any significant time-course change of Thy-1 expression at the transcriptional level (Figure 1D). Thus, acute injury following single-dose bleomycin induced a transient loss of Thy-1 mostly at the surface protein level in activated fibroblasts, likely via shedding or recycling (18). It seems that this transient change in Thy-1 surface expression did not affect myofibroblast disappearance and fibrosis resolution.

Repetitive microinjury resulted in Thy-1 silencing in myofibroblasts, associated with continuous fibrotic remodeling. Next, we used a repetitive lung injury model to determine whether progressive, nonresolving fibrosis is associated with sustained Thy- 1 loss in fibroblasts. Col-GFP mice were subjected to 1 unit/kg bleomycin or saline (control) that was instilled i.t. every 12 days for 4 doses. After 28 or 56 days following the final instillation of bleomycin, lungs were evaluated by measuring Thy- 1 expression, $\mathrm{GFP}^{+}$and/or $\alpha \mathrm{SMA}^{+}$cells, and profibrotic gene expression (Figure $2 \mathrm{~B}$ ). We found that Thy-1 immunostaining obviously disappeared in $\mathrm{GFP}^{+}$fibroblasts or $\mathrm{GFP}^{+} / \alpha \mathrm{SMA}^{+}$myofibroblasts (data not shown) at day 28 and remained low or decreased further at day 56 after final bleomycin challenge (Figure 2, A and C). At both time points, Thy $1 \mathrm{mRNA}$ expression in lung tissue was downregulated in a time-dependent manner (Figure 2D). Moreover, changes in Thy-1 were accompanied by persistent accumulation of $\mathrm{GFP}^{+}$and/or $\alpha \mathrm{SMA}^{+}$(data not shown) cells and continuous fibrotic remodeling, as measured by profibrotic genes Col1 1 and Col3 1 in lung tissue by qPCR (Figure 2D). Together, these findings indicate that repetitive microinjury could induce Thy 1 downregulation or gene silencing, in addition to loss at the protein level, associated with progressive fibrotic tissue remodeling. Thus, the emergence of persistently Thy-1fibroblasts may be an important event that predisposes the lung to compromised and profibrotic repair. 
A
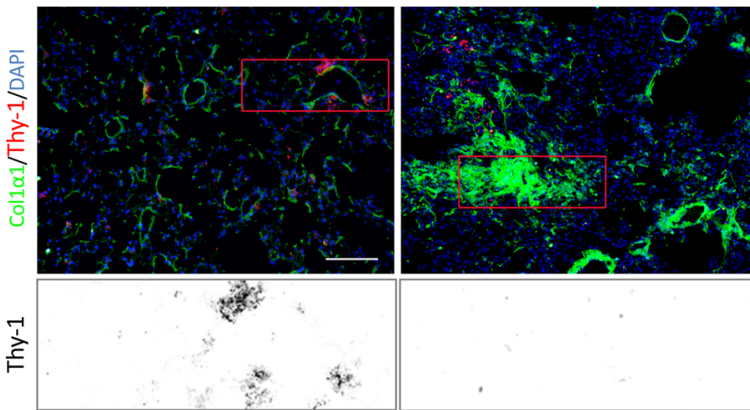

$\mathrm{d} 28$

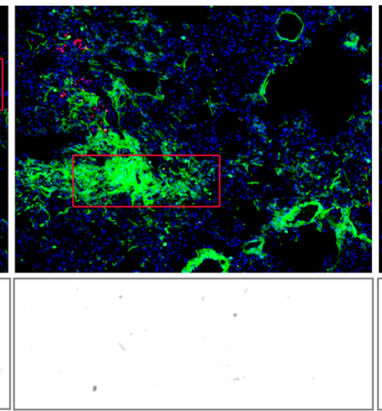

d56

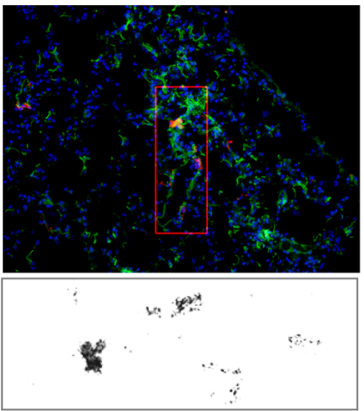

B

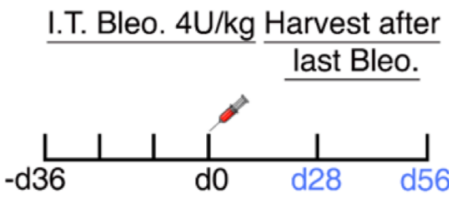

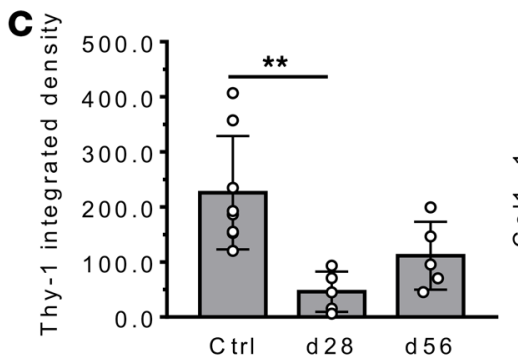
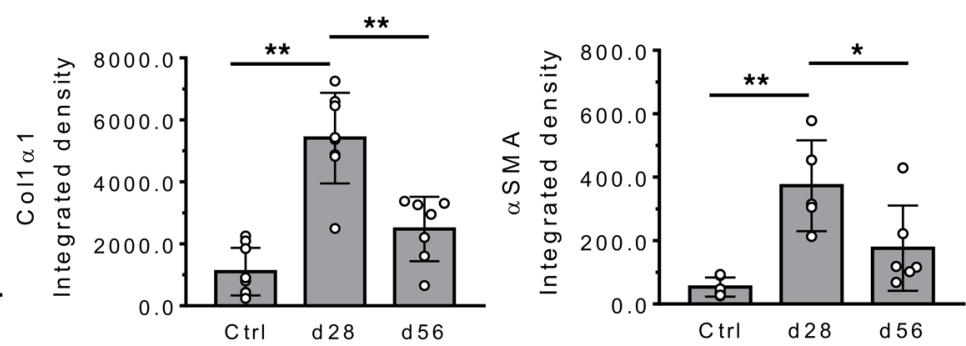

D
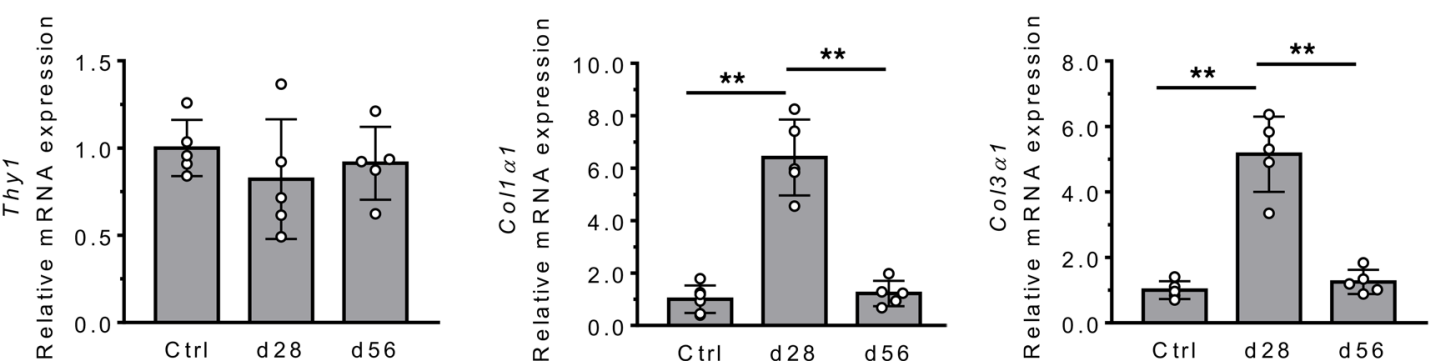

Figure 1. Transient Thy1 loss in GFP' fibroblasts in a self-resolving model of lung fibrosis induced with single-dose bleomycin. (A) In immunofluorescence images of lungs, Col1a1-GFP, Thy-1, and nuclei are overlaid for the entire viewing field. A magnified view of Thy1 is shown. Scale bar: $100 \mu \mathrm{m}$. (B) Experimental scheme. Adult Col1 $\alpha 1$-GFP mice ( $n=5-7$ /group) were given bleomycin (Bleo) ( $4 \mathrm{U} / \mathrm{kg}$ in $100 \mu \mathrm{L}$ saline) by orotracheal intubation (MicroSprayer). Lungs were collected at 28 or 56 days after Bleo instillation. (C) The areas stained positively for Thy-1, Col1 $\alpha 1$, and $\alpha$ SMA were quantified as a total density using ImageJ. (D) mRNA expression of Thy1 and fibrogenic genes (Col/ $\alpha 1$ and Co/3 $\alpha 1$ ) was determined by qPCR. Results are presented as mean \pm SEM. Statistical analysis was performed using 1-way ANOVA; ${ }^{*} P<0.05,{ }^{* *} P<0.01$.

Thy-1 loss is associated with elevated $\alpha v$ integrin activity in vivo and progressive, nonresolving fibrosis. To determine if Thy-1 loss is associated with $\alpha \mathrm{v}$ integrin activation in lung fibrosis, we carried out a time-course study in Thy $1^{-/-}$and WT mice following single-dose bleomycin (Figure 3B). We have previously shown that, Thy $1^{-/-}$ mice, unlike WT mice, fail to resolve fibrosis by 56 days after injury (27). As predicted by Thy-1's role in regulating $\alpha \mathrm{v}$ integrin, we observed sustained $\alpha \mathrm{v} \beta 3$ integrin activation in $\alpha \mathrm{SMA}^{+}$myofibroblasts (indicative of active fibrosis) at all time points through 56 days, as determined by double immunofluorescence (IF) (Figure $3, \mathrm{~A}, \mathrm{C}$, and D). This finding is in contrast to that in WT mice that displayed a reduction in $\alpha \mathrm{v} \beta 3$ integrin staining after 28 days, corresponding to the initiation of resolution of bleomycin-induced fibrosis (Figure 3 , $A$ and $D)$. These findings suggest that persistently activated $\alpha v$ integrin in fibroblasts of mice lacking Thy-1 could result in nonresolving fibrosis. We observed elevated $\alpha \mathrm{v}$ integrin activity at baseline in untreated Thy $1^{-1-}$ lungs, but it was not sufficient to spontaneously induce fibrosis. In the acute injury phase (3 days after bleomycin administration), there were no differences in histopathological alteration (H\&E staining) and lung permeability (total proteins, total counts, and differentials in bronchoalveolar lavage fluid) in WT mice as compared with those in Thy $1^{-1-}$ mice (Supplemental Figure 1; supplemental material available online with this article; https://doi.org/10.1172/jci.insight.131152DS1), suggesting that differences in early inflammation do not account for the differences in the later fibrosis. Collectively, these data demonstrated that the lung milieu in mice lacking Thy-1 promoted persistent activation/upregulation of $\alpha \mathrm{v} \beta 3$ integrin in fibroblasts, resulting in persistence of profibrotic myofibroblast phenotypes in vivo. 
A

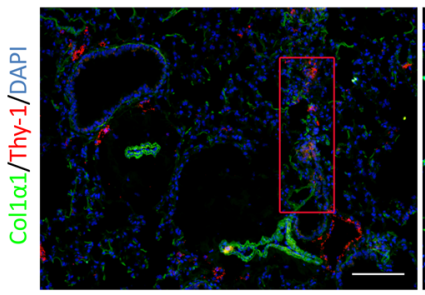

$\mathrm{d} 28$

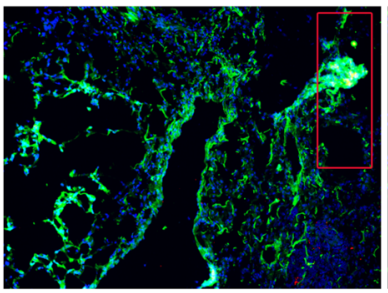

d56

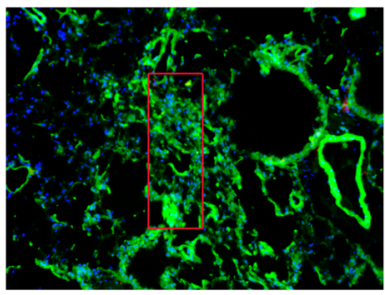

B

$\underline{\text { Repetitive Bleo. }} \underline{\text { Harvest after }}$

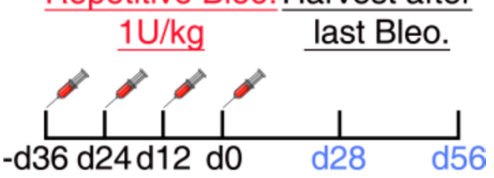

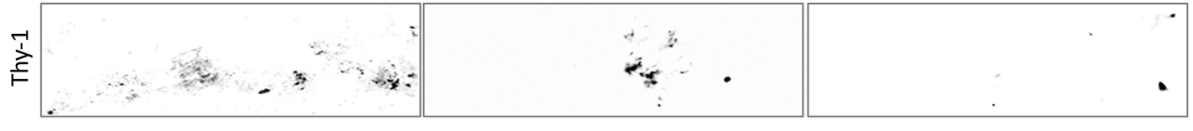
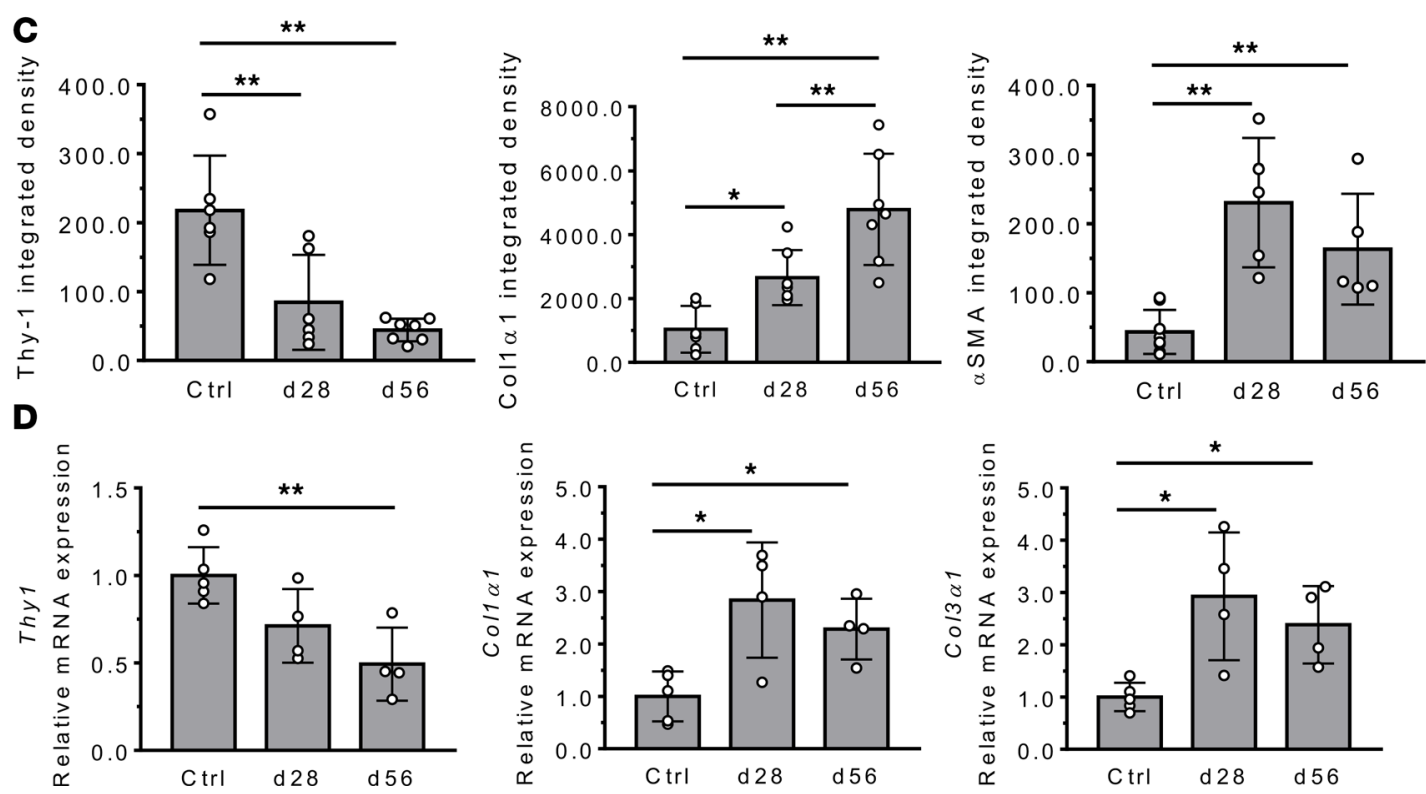

Figure 2. Persistent Thy-1 loss associated with silenced expression in GFP+ fibroblasts in the nonresolving mode of lung fibrosis induced with repeated bleomycin. (A) In immunofluorescence images of lungs, Col1 1-GFP, Thy-1, and nuclei are overlaid for the entire viewing field. A magnified view of Thy-1 is shown. Scale bar: $100 \mu \mathrm{m}$. (B) Experimental scheme. Adult Col1 $\alpha 1$-GFP mice ( $n=5-7 /$ group) were given with bleomycin (Bleo) $(1 \mathrm{U} / \mathrm{kg})$ in $100 \mu \mathrm{L}$ saline, every 12 days, for 4 times by orotracheal intubation (MicroSprayer). Lungs were collected at 28 or 56 days after last Bleo instillation. (C) The positively stained area was quantified as total density using Image). (D) mRNA expression of Thy1 and fibrogenic genes (Col1 $\alpha 1$ and Col3 $\alpha 1$ ) was determined by qPCR of lung tissue. Results are presented as mean \pm SEM. Statistical analysis was performed using 1-way ANOVA; ${ }^{*} P<0.05,{ }^{* *} P<0.01$.

sThy-1 promotes resolution of bleomycin-induced fibrosis. To examine the potential therapeutic effect of a soluble form of human Thy-1 (sThy-1) in lung fibrosis, we gave 1,000 ng/kg sThy-1-IgG Fc or saline control at day 14 after single-dose i.t. bleomycin instillation (i.e., after fibrosis was established) in WT mice and assessed fibrotic parameters at day 21 (Figure 4A). Histopathological examination showed that there was an obvious shift from a much more uniform fibrotic response to limited patchy fibrosis in mice receiving sThy-1-IgG Fc (Figure 4B). The treatment of the mice with sThy-1-IgG Fc significantly reduced collagen deposition (Figure $4, \mathrm{~B}$ and $\mathrm{D})$ and fibrosis scores $(P<0.05$, Figure $4 \mathrm{C})$ when compared with mice treated with bleomycin and PBS. IHC for $\alpha$ SMA (Figure 4B) followed the same patterns of alteration as those seen with histopathological examination, indicating reduction in myofibroblasts or reversal of the myofibroblast phenotype by sThy-1IgG Fc. Administration of sThy-1-IgG Fc in saline-instilled control mice had no effect on lung histology or on the fibrotic and inflammatory endpoints (data not shown). Thus, exogenous sThy-1 may have significant therapeutic potential in promoting resolution of established pulmonary fibrosis.

Targeting of $\alpha v$ integrin by sThy-1 reverses myofibroblast differentiation in a phenotypic in vitro model of IPF fibroblasts. Because IPF fibroblasts may be senescent and resistant to phenotype changes, we tested the effects of sThy-1 on senescent-like myofibroblast differentiation in vitro (30). Briefly, we induced myofibroblast differentiation in human lung fibroblasts (CCL-210) by incubation with recombinant human TGF- $\beta 1$ (10 $\mathrm{ng} / \mathrm{ml}$ ) for 48 hours and, subsequently, senescence by culturing for an additional 5 days in serum-free media 
A
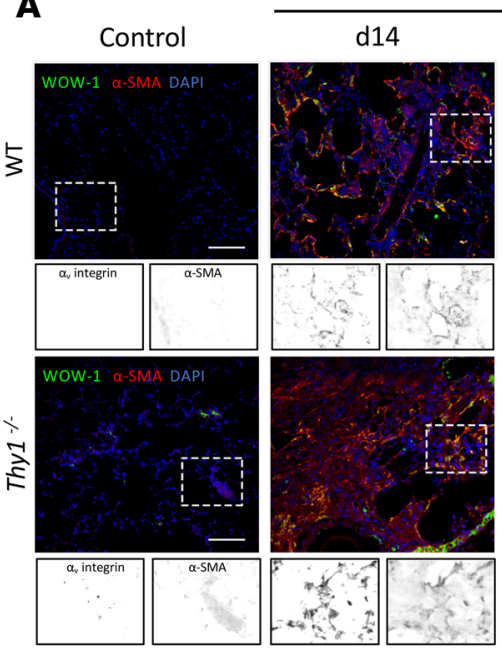

C

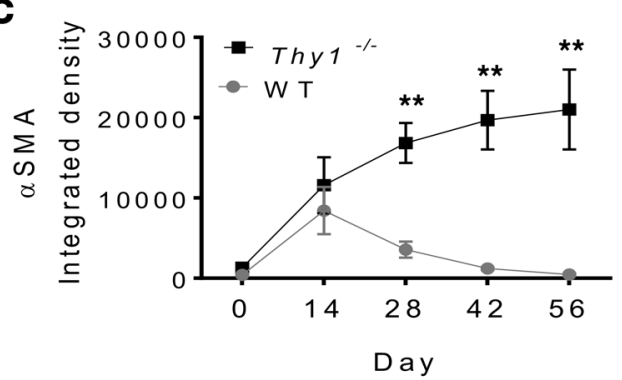

Bleo

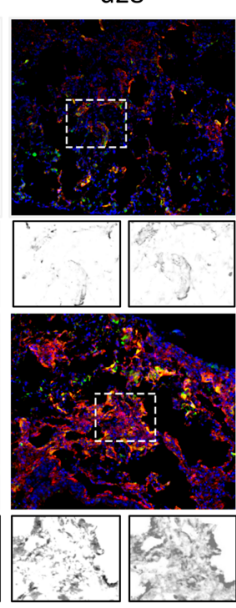

d56

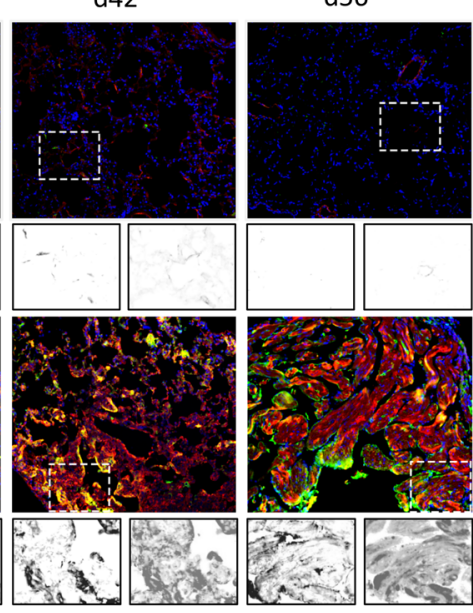

\section{B}

Thy $1^{-/-}$vs. WT

Bleo 4U/kg i.t.

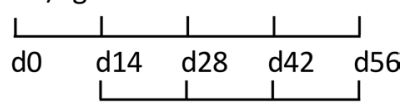

Lung collection

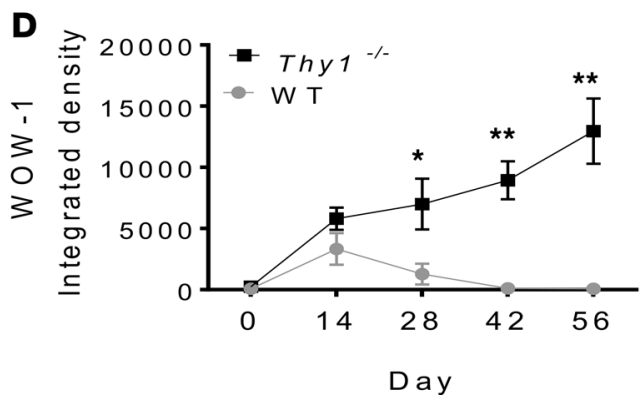

Figure 3. $\alpha \mathbf{v} \boldsymbol{\beta} 3$ Integrin is persistently activated or upregulated in mice lacking Thy-1 expression. (A) Immunofluorescence (IF) for $\alpha \mathrm{V}$ integrin and $\alpha$ SMA in lung tissue. (B) Experimental scheme. Adult WT and Thy 1/- mice were given single-dose bleomycin (Bleo) (4 U/kg, in 100 $\mu \mathrm{L}$ saline) by orotracheal intubation (MicroSprayer). Lungs were collected at 14, 28, 42, and 56 days after Bleo instillation ( $n=4-5 /$ group). (C and D) IF quantification of active $\alpha v \beta 3$ integrin (WOW-1) and $\alpha$ SMA in lung tissue. Scale bar: $100 \mu \mathrm{m}$. Results are presented as mean \pm SEM. Two groups were compared using 2-tailed Student's $t$ test, ${ }^{*} P<0.05$, ${ }^{* *} P<0.01$ versus WT group.

to control proliferation. Context-dependent senescence (as evidenced by p21 expression) was induced in TGF- $\beta 1$-treated and serum-fasted fibroblasts (Figure 5A). Thus, this cellular model may be more appropriate to represent phenotypes of IPF fibroblasts. Then, we treated these cells with recombinant human sThy-1-IgG Fc (10, 100, 1,000 ng/mL), RLE-mutated Thy-1 [Thy-1(RLE)-IgG Fc] (1000 ng/mL), and IgG Fc $(1000 \mathrm{ng} / \mathrm{mL})$ for 48 hours. As determined by qPCR of Acta 2 and Coll $\alpha 1$ and immunoblotting of $\alpha$ SMA expression, addition of sThy-1-IgG Fc at the $1000 \mathrm{ng} / \mathrm{mL}$ level significantly reduced myofibroblastic differentiation when compared with TGF- $\beta 1$, PBS control (Figure 5, B-D; see complete unedited blots in the supplemental material). These changes suggest that exogenous sThy- 1 is effective at reversing myofibroblast differentiation, even in senescent-like cells. Because the compounds all showed some nonspecific effects (although not statistically significant), the difference between sThy-1(RLE) and sThy-1 shows a reduced trend, suggesting that the RGD-like integrin-binding motif in Thy-1 is required.

sThy-1 reverses established TGF- $\beta 1$-induced lung fibrosis in mice. To determine the therapeutic potential of sThy-1 in an alternative, TGF- $\beta 1$-driven genetic model of fibrosis, we investigated the effect of human sThy-1 on doxycycline-induced (Dox-induced) expression of active human TGF- $\beta 1$, which drives lung fibrosis in Cc10-rtTA-tTS-Tgfb1 $\mathrm{Tg}^{+}$mice (Figure 6B). Twenty-eight days after Dox induction, Cc10-rtTA$t T S-T g f b 1 \mathrm{Tg}^{+}$mice showed apparent airway and alveolar parenchymal fibrotic response, characterized by alveolar septal thickening and areas of septal rupture, as determined by histopathological analyses (H\&E and Trichrome staining, Figure 6A). Treatment of these mice at day 28 with $1000 \mathrm{ng} / \mathrm{kg}$ sThy-1-IgG Fc i.v., but not sThy-1(RLE) or IgG, showed resolution of airway and alveolar parenchymal fibrotic response (Figure 6A). Administration of sThy-1 resulted in a significant reduction in the level of hydroxyproline (Figure 6C), expression of $\alpha \mathrm{v}$ integrin, murine TGF- $\beta 1$, and $\alpha \mathrm{SMA}$ measured by IF (Figure 6D), and reduction in expression of profibrotic genes (Colla1, Col3 $\alpha 1$, Tgfb1, and Acta2) by qPCR (Figure 6E). 
A
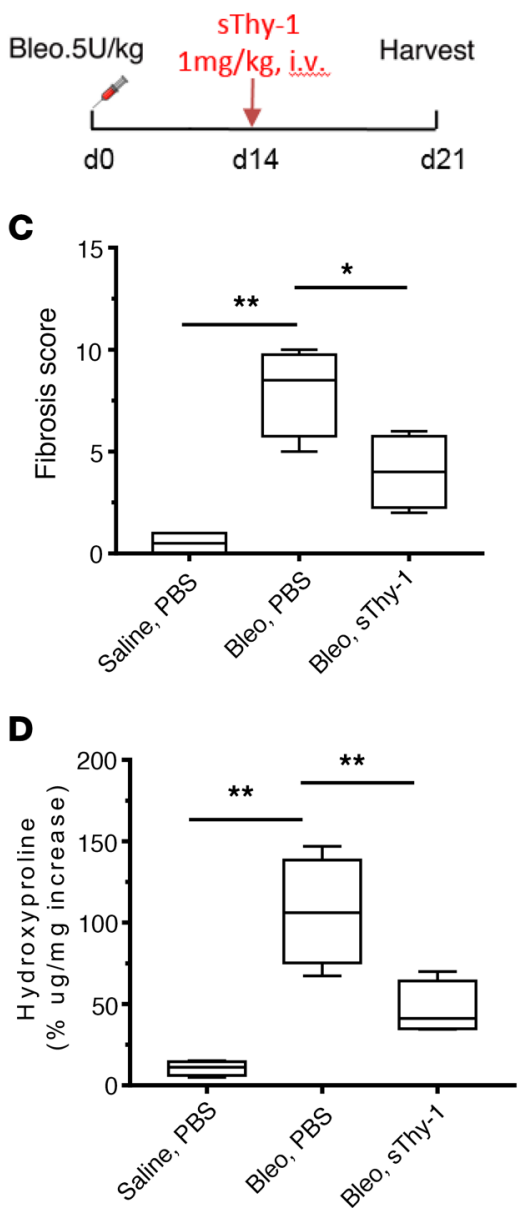

B

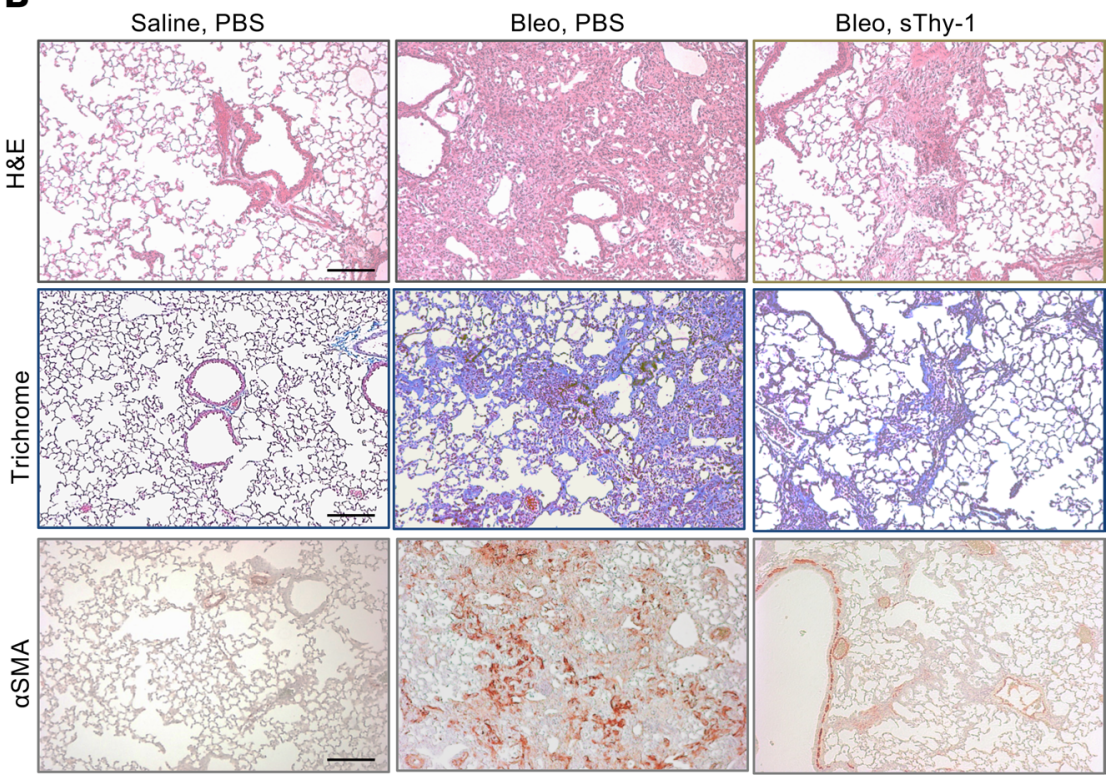

Figure 4. Soluble Thy-1 reverses established bleomycin-induced lung fibrosis in mice. (A) Dosing regimen in a single-dose i.t. bleomycin (Bleo) model of lung fibrosis: human sThy1-Fc (1 mg/kg) or PBS was given i.v. in C57BL/6 WT mice at day 14 after $5 \mathrm{U} / \mathrm{kg}$ Bleo treatment by orotracheal intubation (MicroSprayer) ( $n=5$ /group). At day 21 , lungs were collected and $10 \%$ formalin fixed, paraffin embedded, and processed. (B) H\&E, Masson's trichrome, and IHC staining of $\alpha$ SMA. Scale bar: $200 \mu \mathrm{m}$. (C) Fibrosis score was calculated using H\&E-stained slides. (D) Half lungs were used for quantification of hydroxyproline content. Results are presented as mean \pm SEM. Statistical analysis was performed using 1-way ANOVA; ${ }^{*} P<0.05$, ${ }^{* *} P<0.01$ versus Bleo-PBS group.

These changes indicate that the exogenous sThy- 1 molecule is effective at reversing myofibroblast differentiation and established fibrosis in vivo, associated with decreased $\alpha \mathrm{v}$ integrin activation. The therapeutic effect of sThy-1-IgG Fc, but not sThy-1(RLE)-IgG Fc, indicates that the Thy-1 RLD is required. Taken together, these data demonstrate that human sThy-1 reverses lung fibrosis via trans regulation of $\alpha \mathrm{v}$ integrin activity in Dox-induced $C c 10-r t T A-t T S-T g f b 1 \mathrm{Tg}^{+}$mice, underscoring its therapeutic potential in resolution of established pulmonary fibrosis in two distinct models of fibrosis.

\section{Discussion}

In these studies, we demonstrate a critical homeostatic role of a primary Thy-1- $\alpha \mathrm{v}$ integrin interaction upstream of canonical ECM-integrin ligation in vivo, disruption of which is associated with progressive fibrogenesis following lung injury. In a severe acute lung injury, such as in the single-dose bleomycin model, loss of Thy- 1 is incomplete and transient, and recovery of Thy- 1 expression occurs during fibrosis resolution. However, repetitive administration of lower doses of bleomycin induced progressive, nonresolving lung fibrosis associated with sustained transcriptional silencing of Thy1 expression in myofibroblasts. Although transient loss of Thy-1 is related to fibroblast activation (22), silencing of Thy1 may initiate a more permanent shift toward a Thy-1 $1^{-}$, profibrotic, apoptosis-resistant myofibroblast phenotype (18). We have previously demonstrated epigenetic silencing of Thy1 expression in lesional fibroblasts in fibroblastic foci of pulmonary fibrosis and demonstrated reversal of myofibroblastic differentiation associated with restored Thy1 expression following epigenetic modifiers in vitro (26). This paradigm has been demonstrated by others in vivo in the context of aging and TGF- $\beta 1$ (31) as well as in the context of sustained TLR4 activation (32).

Additionally, we confirm that sustained absence of Thy-1 in the context of lung injury (such as in Thy $1^{-1}$ mice following bleomycin) is associated with progressive, nonresolving fibrosis (33) and, as we show here, sustained activation of $\alpha \mathrm{v}$ integrin. Thy-1 loss activates $\alpha \mathrm{v}$ integrin, which facilitates myofibroblast differentiation, 
A

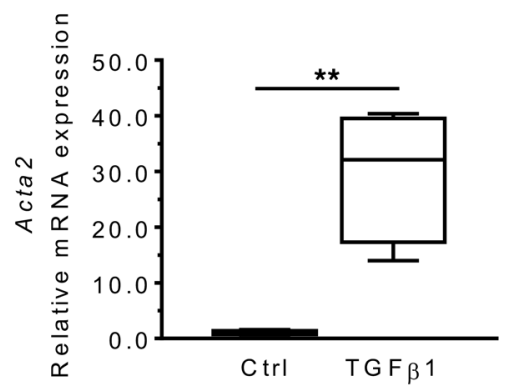

\section{B}

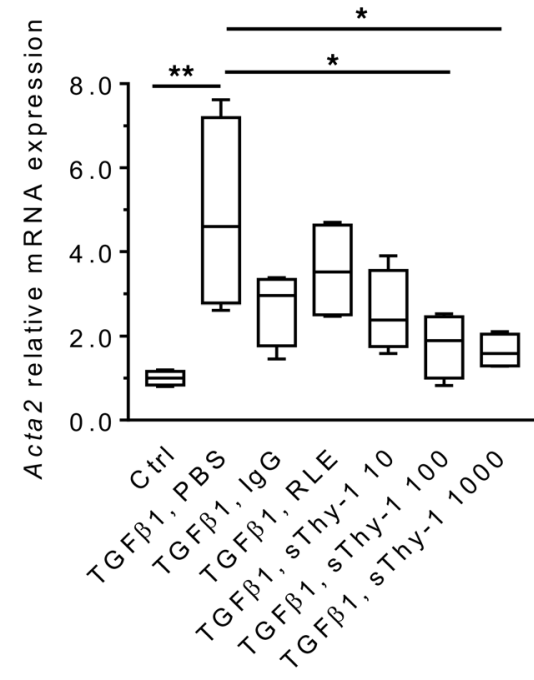

C

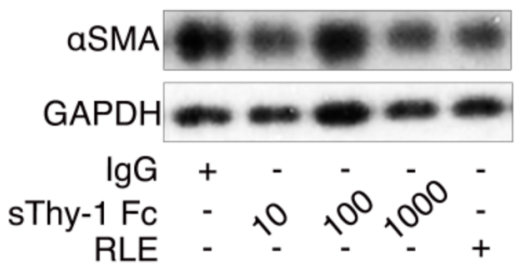

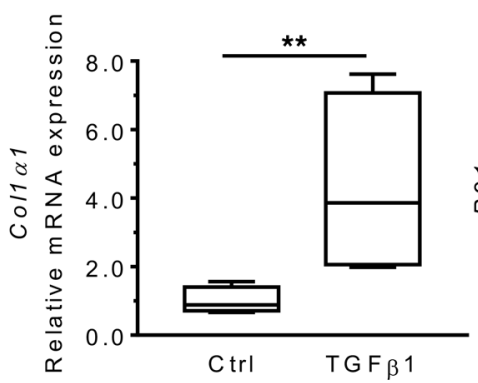
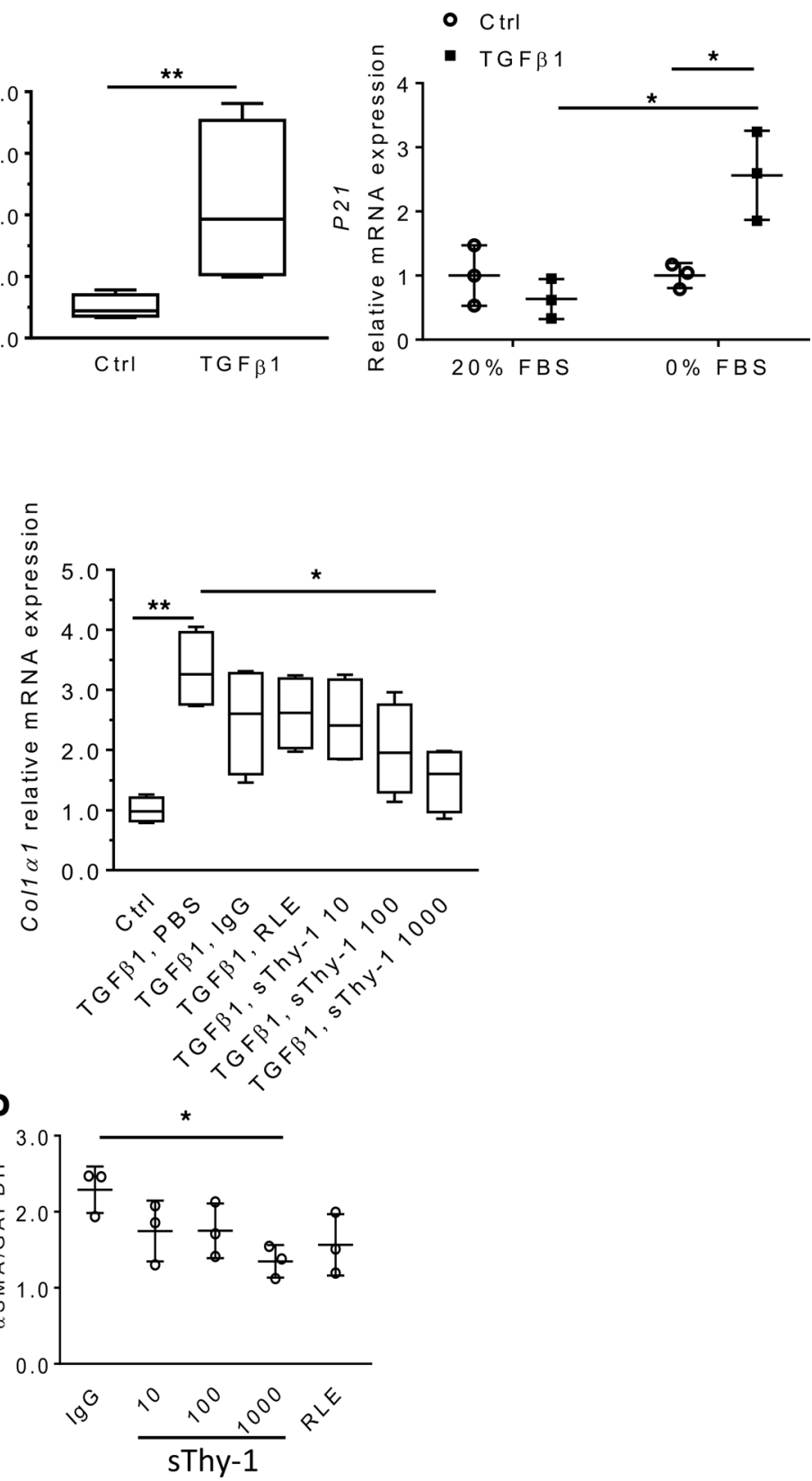

Figure 5. Soluble Thy-1 reverses myofibroblastic differentiation of senescent human lung myofibroblasts in a dose-dependent manner in vitro. Human lung fibroblasts (CCL-210) were incubated with human TCF- $\beta 1$ (10 ng/ml for 48 hours) and subsequently subjected to 5-day serum-free media to induce senescence ( $n=4$ /group, 3 times). (A) Myofibroblast differentiation and senescence were validated by qPCR of Acta2, Col1 $\alpha 1$, and $p 21$. (B) qPCR for Acta2 and Col1 1 after treatment of myofibroblasts with sThy-1-IgG Fc (10, 100, $1000 \mathrm{ng} / \mathrm{mL})$, Thy-1(RLE)-IgG Fc (1000 ng/ml), or lgG Fc (1000 $\mathrm{ng} / \mathrm{ml}$ ) for 48 hours. (C) Western blot (WB) of $\alpha$ SMA expression. (D) WB quantification. Results are presented as mean \pm SEM. Statistical analysis was performed using 2-tailed Student's $t$ test and 1-way ANOVA; ${ }^{*} P<0.05,{ }^{* *} P<0.01$.

likely via multiple downstream pathways. Our data showed that sThy-1 could reverse TGF- $\beta 1$-induced myofibroblast differentiation in vitro, likely via trans-regulation of $\alpha \mathrm{v}$ integrins (34), even in the context of induced senescence. Furthermore, treatment of Dox-induced Cc10-rtTA-tTS-Tgfb1 mice and bleomycin-induced mice with sThy-1 demonstrated antifibrotic efficacy. Collectively, these findings suggest that targeting of av integrin by sThy-1 molecules could be a novel strategy to treat lung fibrosis, possibly halting the progression or speeding the resolution of lung fibrosis.

Our previous in vitro studies indicated the potential of sThy-1, via integrin engagement, to reverse the experimental induction of the myofibroblastic phenotype (27), but its antifibrotic efficacy in vivo, where fibrosis is a more complex pathophysiologic process, had not been previously determined. To this end, 


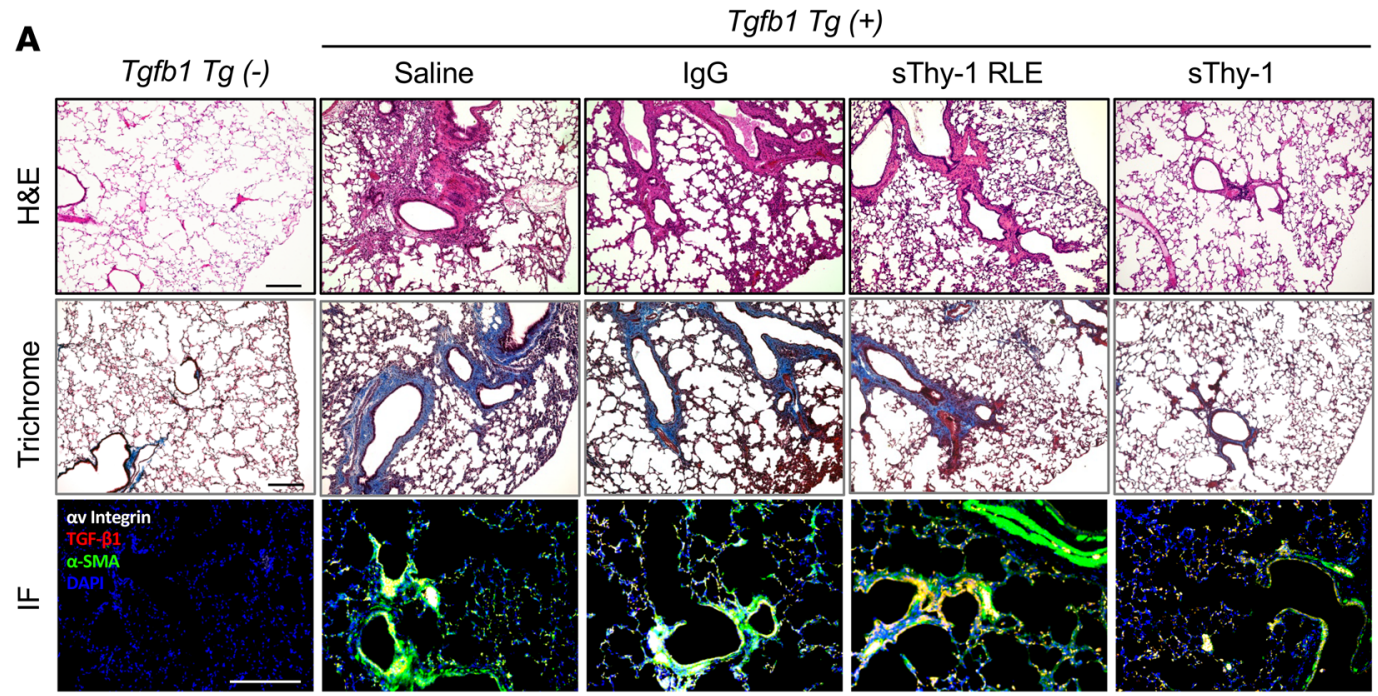

$B$

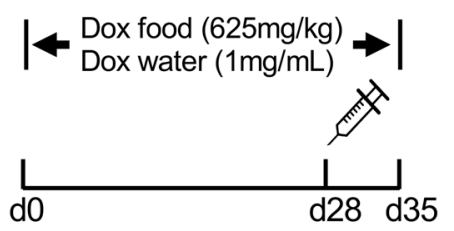

c

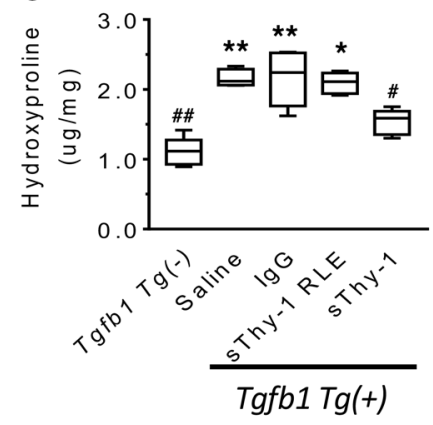

D
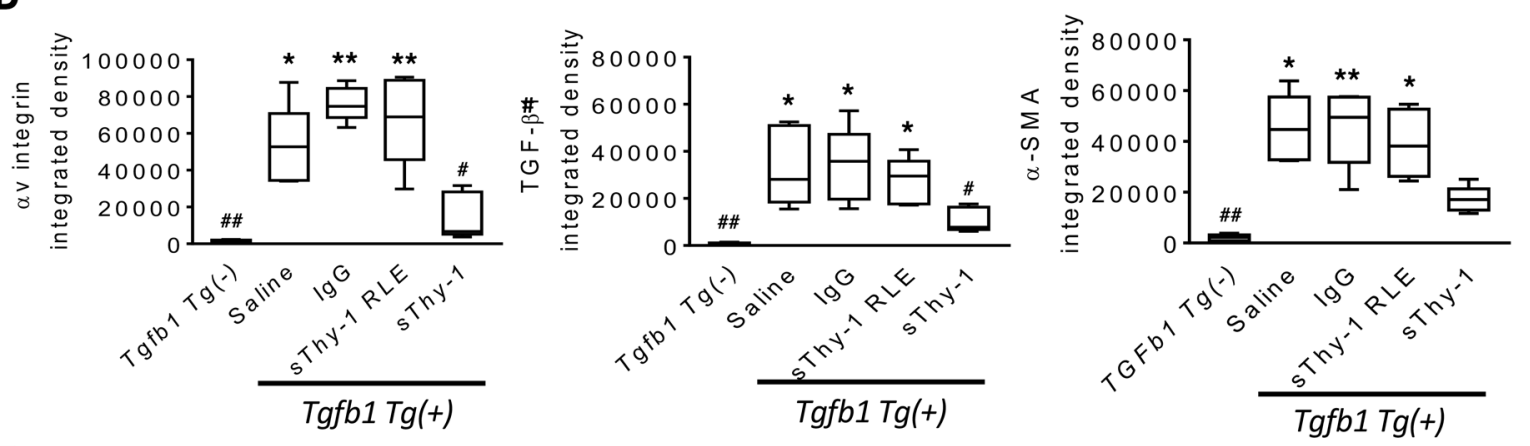

E
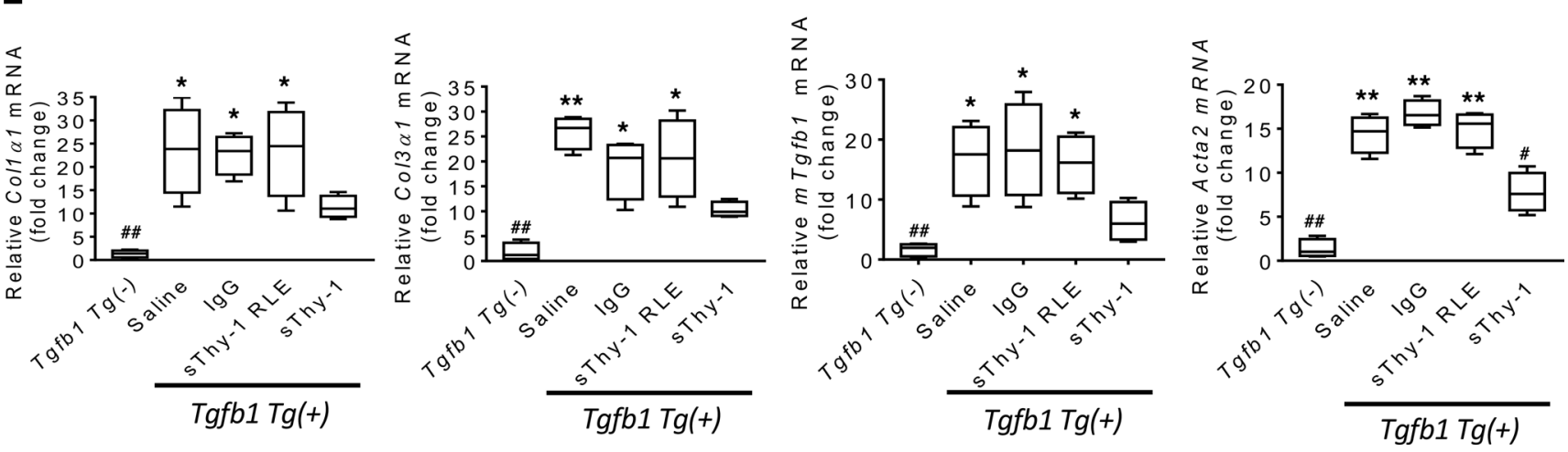

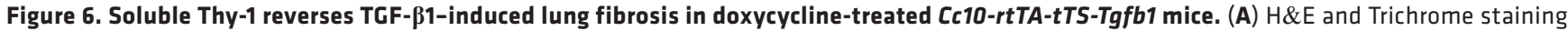
and immunofluorescence (IF) for $\alpha v$ integrin, $\alpha$ SMA, and TGF- $\beta 1$ in lung tissue. Scale bar: $200 \mu \mathrm{m}$. (B) Mice were randomized to doxycycline (Dox) in food $(625 \mathrm{mg} / \mathrm{kg})$ and water $(1.0 \mathrm{mg} / \mathrm{mL})$ for 4 weeks. Dox-induced mice were treated i.v. with a single $1 \mathrm{mg} / \mathrm{kg}$ dose of either recombinant human Thy-1-Fc or RLE-mutated Thy-1 [Thy-1(RLE)-IgG Fc], IgG-Fc, or saline at day 28 . The lungs were assessed 1 week after treatment ( $n=4-5 / g r o u p)$. 
(C) Hydroxyproline quantification in lung tissue. (D) Quantification of $\alpha v$ integrin, $\alpha$ SMA, and TGF- $\beta 1$ IF staining. (E) Profibrotic genes (Col1 $\alpha 1$, Col3 $\alpha 1, T g f b 1$, and Acta2) in lung tissue extracts by qPCR. Results are presented as mean \pm SEM. Statistical analysis was performed using 1-way ANOVA; ${ }^{*} P<0.05,{ }^{* *} P<0.01$ versus sThy-1 group; ${ }^{\#} P<0.01$ versus Saline, IgG, and sThy-1(RLE) groups; $P<0.05$ versus Tgfb1 Tg- group.

we examined the effects of exogenously administered sThy-1 on established lung fibrosis (14 days after bleomycin). The data clearly support the therapeutic effect of sThy-1 in promoting resolution of fibrosis, especially indicating reduction in myofibroblasts (or reversal of the myofibroblast phenotype) by sThy-1. The beneficial effect of a single treatment with sThy-1 suggests a "reset" of the fibrogenic program. Because single-dose bleomycin-induced fibrosis is self-resolving, sThy-1 in this context may act to facilitate or accelerate endogenous programs for resolution. In order to test the therapeutic potential of sThy- 1 in a more progressive model, we selected the $C c 10-r t T A-t T S-T g f b 1$ mice, in which transgene induction with Dox results in expression of the active form of human TGF- $\beta 1$, which initiates a self-sustaining fibrogenic milieu, and we chose to intervene a later time point (day 28) when fibrotic remodeling is well established. Remarkably, a single administration of sThy-1 promoted significant resolution of fibrosis over the ensuing 7 days, with improvement in multiple histologic and biochemical measures of fibrosis, including expression of endogenous TGF- $\beta 1$. The effect is dependent on the Thy- 1 RLD sequence and accompanied by decreased activation of $\alpha \mathrm{v}$ integrin, suggesting engagement of an important homeostatic axis. The cellular and molecular mechanisms of this effect are likely to be complex but could involve restoration of mechanosensitive signaling (29), myofibroblast apoptosis (18), and interruption of latent TGF- $\beta 1$ activation $(22,35)$. The modulation of fibroblast senescence may possibly be involved in the Thy-1//integrin/TGF- $\beta 1$ pathway (34). It is unknown whether trans-signaling from exogenous sThy-1 alters epigenetic mechanisms regulating Thy1 expression.

Thy-1 is a complex biological molecule, with a variety of functions in multiple cell types (36). In addition to the RLD integrin-binding motif, Thy-1 also has domains that interact with heparan sulfate proteoglycans, and the degree to which these additional interactions are important for its antifibrotic effects is unclear, although a single amino acid change in the RLD was sufficient to abrogate the effects. It is also unclear the degree to which the effects of sThy-1 differ from other av-targeted entities.

This study has important implications for therapeutic development. Most antifibrotic agents limit ongoing fibrogenesis but do not promote resolution of existing fibrosis. sThy-1 seems to have the latter activity and may be useful in a disease such as IPF, which displays temporal heterogeneity, with areas of active fibrogenesis alternating with areas of more established fibrosis. The fibrogenic phase of acute lung injury, which has the capacity to resolve but also has an extremely high mortality rate and is subject to epigenetic alterations that promote progression, may benefit from the proresolution "reset" activity seen in our studies. There is significant enthusiasm for cell-based therapies for acute lung injury and fibrosis, many of which use mesenchymal stem cells (which are strongly Thy- $1^{+}$) or their products to restore a homeostatic regenerative milieu. Experts advise tempering such enthusiasm with appropriate caution (37), as cell-based regenerative therapies are complex and may have unanticipated long-term consequences. sThy-1 may be able to mediate some of the beneficial effects of cell-based therapies with fewer concerns, especially if it can be administered in a single administration or a series of intermittent infusions (Figure 7). In any case, the dramatic proresolution effects seen in two different models, and supported by decades of mechanistic understanding, warrant further study to assess the translational potential of sThy-1 as antifibrotic therapy.

\section{Methods}

Cell culture. Human lung fibroblasts (CCL-210, ATCC) (50,000 cells) were cultured in 6-well plates in Dulbecco's modified Eagle medium (Thermo Fisher Scientific, 11960-044), 10\% fetal bovine serum, and $1 \%$ penicillin-streptomycin (Thermo Fisher Scientific, ICN1670249). Confluent cells were passaged by $0.25 \%$ trypsin. To rule out serum effects, fibroblasts were incubated in serum-deprived media for 16 hours prior to TGF- $\beta 1$ treatment. By treating with TGF- $\beta 1$ in serum-free media $(10 \mathrm{ng} / \mathrm{mL})$ for 48 hours, differentiation into myofibroblasts was induced. TGF- $\beta 1$-induced myofibroblasts were subjected to FBS $0 \%$ or $20 \%$ media to model myofibroblast senescence. Then, cells were treated with indicated concentration of soluble recombinant human Thy-1 Fc (10, 100, $1000 \mathrm{ng} / \mathrm{mL})($ Enzo Life Science, ALX-522-091-C050), human IgG-FC (1000 ng/mL) (Enzo Life Science, ALX-203.005.2060), Thy-1(RLE)-FC (1000 ng/mL) (Enzo Life Science, ALX-522-097-C050), or PBS for 48 hours to test 


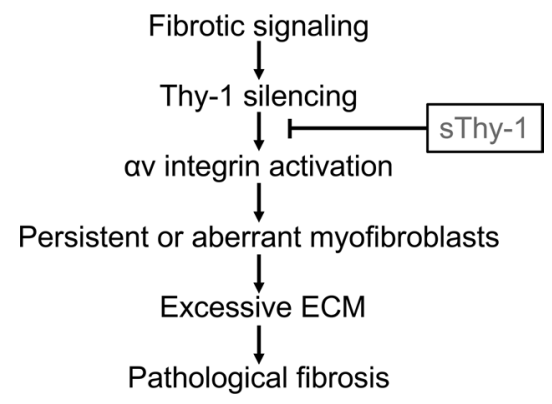

Figure 7. The proposed pathway of fibroblastic Thy-1 silencing and sThy-1 addition in lung fibrosis.

dedifferentiation from myofibroblasts. sThy-1-FC, human IgG-FC, and sThy-1(RLE)-FC were reconstituted in PBS before use. Cells without TGF- $\beta 1$ treatment were used as a negative control.

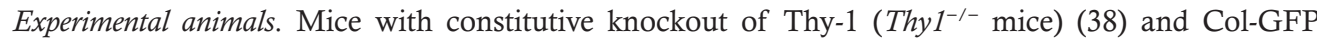
[expressing collagen-a1(I) promoter/enhancer-driven GFP] mice have been previously described (5). Thy $1^{-/-}$Col-GFP mice were generated by crossing of Thy $1^{-/-}$and Colla1-GFP mice. Cc10-rtTA-tTS-Tgfb1 mice (a gift of Erica Herzog, Yale University, New Haven, Connecticut, USA), a triple-transgenic model, allow bioactive hTGF- $\beta 1$ to be expressed conditionally in the lung via Dox induction (35). Genotyping of all mice was performed by PCR. WT C57/BL6 mice were purchased from The Jackson Laboratory.

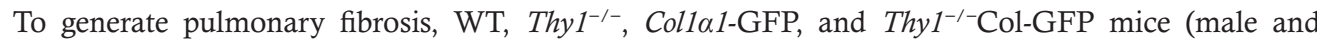
female, 24 weeks old) were anesthetized with intraperitoneal injections of ketamine and xylazine (100 and $10 \mathrm{mg} / \mathrm{kg}$ body weight, respectively). Mice were given a single $(4 \mathrm{U} / \mathrm{kg})$ or repetitive $(1 \mathrm{U} / \mathrm{kg}$, every 10 days for 4 doses) i.t. instillation of bleomycin sulphate ( $4 \mathrm{U} / \mathrm{kg}$ body weight, dissolved in $100 \mu \mathrm{L}$ sterile saline, McKesson) or sterile saline on day 0 using MicroSprayer MS-IA-1C (Penn-Century). Following the instillation, mice were allowed to be monitored daily for mortality. To conditionally induce pulmonary fibrosis, Cc10-rtTA-tTS-Tgfb1 mice (male and female, 24 weeks old) were randomized to food containing Dox (625 $\mathrm{mg} / \mathrm{kg}$ ) and water with Dox $(1.0 \mathrm{mg} / \mathrm{mL})$ or normal food and water for 4 weeks (35). All adult mice were housed in the Association for Accreditation and Assessment of Laboratory Animal Care-approved animal facility at the University of California San Diego School of Medicine.

$s$ Thy-1 treatment. Dox- and bleomycin-induced mice were treated i.v. with $1000 \mu \mathrm{g} / \mathrm{kg}$ of either soluble recombinant human Thy-1-Fc (Enzo Life Science, ALX-522-091-C050) or Thy-1(RLE)-IgG Fc (Enzo Life Science, ALX-522-097-C050), IgG-FC (Enzo Life Science, ALX-203.005.2060), or with saline. Lungs were assessed 1 week after treatment by histopathology (H\&E and Trichrome staining) and collagen content (measuring hydroxyproline), and profibrotic genes Coll $\alpha 1$, Col3 $\alpha 1$, and $m T g f b 1$ were measured in the lung tissue extracts by qPCR. Expression of $\alpha \mathrm{SMA}$ and $\alpha \mathrm{v}$ integrin in lung slides was determined by IF.

Histopathological analysis. Lungs were fixed (10\% formalin at a constant pressure of $\left.20 \mathrm{~cm} \mathrm{H}_{2} \mathrm{O}\right)$ and paraffin embedded. Five- $\mu \mathrm{m}$-thick sagittal sections of all lobes of fixed lungs were cut and subsequently stained with $H \& E$ and Masson's Trichrome to evaluate histopathologic changes. Severity of fibrosis was quantified from H\&E-stained entire lungs using the Ashcroft scoring system (39). The degree of fibrosis was graded from 0 (normal lung) to 8 (severe distortion of structure, large fibrous areas, and honeycomb lesions). The mean score from all fields (magnification, $\times 200$; average 30 fields/animal) was taken as the fibrosis score.

$I F$. Lungs were sectioned at $6 \mu \mathrm{m}$ (paraffin embedding) or $10 \mu \mathrm{m}$ (OCT embedding), followed by double IF with antibodies. Briefly, lung slides were fixed with 3.7\% formaldehyde for 10 minutes, permeabilized with $0.25 \%$ Triton X-100 (Thermo Fisher Scientific) for 8 minutes, and blocked with1\% BSA (Amresco) for 1 hour. Lung sections were incubated at $4^{\circ} \mathrm{C}$ overnight with primary antibodies: Thy-1 (1:300, Bio-Rad, Mca1474), WOW-1 (1:200, a gift from Sanford Shattil, UCSD), $\alpha$ SMA (1:300, abcam, Ab21027), $\alpha$ v integrin (CD51) (1:200, eBioscience, 12-0512-81), and TGF- $\beta 1$ (1:200, GeneTex, 110630). After the sections were washed with PBS, Alexa Fluor-coupled secondary antibodies (1:1000, Invitrogen), goat anti-rabbit IgG $(\mathrm{H}+\mathrm{L})$, and Texas Red (1:250, Thermo Fisher, T-2767) were used as secondary antibodies. Nuclear staining was carried out with DAPI using ProLong Diamond Antifade Mountant medium (Invitrogen, P36962). Fluorescence images were captured on a BZ-X700 microscope (Keyence). To determine the fluorescence signal in tissue sections, fluorescent cells in 5 randomly different high-power fields from each slide were quantified. 
Hydroxyproline quantification. Hydroxyproline content was measured using the hydroxyproline assay kit from MilliporeSigma (MAK008) according to the manufacture's instruction with slight modification. In brief, whole lungs were homogenized in $\mathrm{dH}_{2} \mathrm{O}$, using $100 \mu \mathrm{L} \mathrm{dH}_{2} \mathrm{O}$ for every $10 \mathrm{mg}$ of tissue. $200 \mu \mathrm{L}$ concentrated $\mathrm{HCl}(6 \mathrm{~N})$ was added to $100 \mu \mathrm{L}$ of tissue homogenate in a pressure-tight, Teflon-capped vial, and the mixture was hydrolyzed at $120^{\circ} \mathrm{C}$ for 3 hours, followed by filtration through a $45-\mu \mathrm{m}$ syringe filter (Millipore). $10 \mu \mathrm{L}$ of the hydrolyzed sample was transferred to a 96 -well plate and was evaporated to dryness under vacuum, to which $100 \mu \mathrm{L}$ Chloramine $\mathrm{T}$ reagent was added per well. After incubation at room temperature for 5 minutes, $100 \mu \mathrm{L}$ p-Dimethylaminobenzaldehyde reagent was added to each well and further incubated for 90 minutes at $60^{\circ} \mathrm{C}$. Absorbance was measured at $560 \mathrm{~nm}$ in a microplate reader (SpectroMax Plus384).

Western blot. Cultured cells were homogenized in RIPA tissue lysis buffer plus protease and phosphatase inhibitor cocktail (Thermo Fisher Scientific). Equal protein amounts of each lysate were separated on SDS-polyacrylamide gels by electrophoresis before being transferred to polyvinylidene difluoride membranes by electroblotting. Membranes were blocked in TBST with 5\% nonfat dry milk and incubated with antibodies $\alpha$ SMA (1:1000, EMD Millipore, Mabt381) and GAPDH (1:1000, Cell Signaling Technology, $14 \mathrm{C} 10$ ) at $4^{\circ} \mathrm{C}$ with constant rocking overnight. Bound primary antibodies were visualized using HRP-linked anti-rabbit IgG (1:3000, Cell Signaling Technology, 7074) with conjugated horseradish peroxidase and enhanced chemiluminescence reagent. Protein bands were semiquantified using ImageJ Software (NIH).

Quantitative RT-PCR analysis. Lung lobe was homogenized in $1 \mathrm{~mL}$ Trizol reagent (Invitrogen, Thermo Fisher Scientific). Total RNA was isolated and cDNA synthesized commercially (Bio-Rad). Real-time RT-PCR was performed using iTaq Universal SYBR Green Supermix (Bio-Rad) and using the CFX96 real-time PCR detection system (Bio-Rad). Primers were designed by using Beacon Designer (Bio-Rad) and listed in Supplemental Table 1. Assays for each sample and primer set were performed in duplicate, with each reaction using $20 \mathrm{ng} \mathrm{cDNA}$ and $10 \mu \mathrm{M}$ primers in a total reaction volume of $20 \mu \mathrm{L}$. Thermal cycling conditions were $95^{\circ} \mathrm{C}$ for 30 seconds and 35 cycles of $95^{\circ} \mathrm{C}$ for 45 seconds, $57^{\circ} \mathrm{C}$ for 30 seconds, and $72^{\circ} \mathrm{C}$ for 60 seconds. The relative quantification of gene expression was determined using the comparative CT method. Actin or $\mathrm{B} 2 \mathrm{~m}$ was used to normalize the expression data. Comparative threshold $(\Delta \Delta \mathrm{Ct})$ was calculated to determine the changes of gene. Individual lung cDNA samples $(n=3-6)$ were analyzed for the relative expression of mouse Thy1, Col1 $\alpha 1$, Col3 $\alpha 1$, Acta2, and Tgfb1.

Statistics. Statistical analysis was performed using GraphPad Prism 7.0. Values are expressed as mean \pm SEM. The statistical differences were calculated using 2-tailed Student's $t$ test between 2 groups and 1-way ANOVA for multiple comparisons. $P<0.05$ was considered statistically significant.

Study approval. All procedures involving animals were approved by the UCSD Institutional Animal Care and Use Committee, which serves to ensure that all federal guidelines concerning animal experimentation are met.

\section{Author contributions}

SSW and JSH conceptualized and designed the project. SSW, CT, and MJ conducted these experiments and data analysis. CRE, CT, CK, XL, and EC assisted with experiments. SSW and CT wrote the manuscript, with edits from JSH. SSW conceptualized and oversaw the project and drafted the initial manuscript, and CT made most of the figures and did the bulk of the revisions.

\section{Acknowledgments}

We would like to acknowledge the Neuroscience Light Microscopy Core (NIH P30 NS 047101) at UCSD for their assistance. This project was supported by NIH grants HL082818 and HL111169 to JSH.

Address correspondence to: James S. Hagood, Pediatrics (Pulmonology), University of North Carolina at Chapel Hill, 450 MacNider, CB\# 7217, 333 S. Columbia St., Chapel Hill, North Carolina 27599-7217, USA. Phone: 919.966.1055; Email: jhagood@unc.edu. 
1. King TE, et al. A phase 3 trial of pirfenidone in patients with idiopathic pulmonary fibrosis. N Engl J Med. 2014;370(22):2083-2092.

2. Richeldi L, et al. Efficacy and safety of nintedanib in idiopathic pulmonary fibrosis. N Engl J Med. 2014;370(22):2071-2082.

3. Iredale JP, et al. Mechanisms of spontaneous resolution of rat liver fibrosis. Hepatic stellate cell apoptosis and reduced hepatic expression of metalloproteinase inhibitors. J Clin Invest. 1998;102(3):538-549.

4. Desmoulière A, Redard M, Darby I, Gabbiani G. Apoptosis mediates the decrease in cellularity during the transition between granulation tissue and scar. Am J Pathol. 1995;146(1):56-66.

5. Kisseleva T, et al. Myofibroblasts revert to an inactive phenotype during regression of liver fibrosis. Proc Natl Acad Sci U S A. 2012;109(24):9448-9453.

6. Glasser SW, Hagood JS, Wong S, Taype CA, Madala SK, Hardie WD. Mechanisms of lung fibrosis resolution. Am J Pathol. 2016;186(5):1066-1077.

7. Gui YS, et al. mTOR overactivation and compromised autophagy in the pathogenesis of pulmonary fibrosis. PLoS One. 2015;10(9):e0138625.

8. Ho YY, Lagares D, Tager AM, Kapoor M. Fibrosis - a lethal component of systemic sclerosis. Nat Rev Rheumatol. 2014;10(7):390-402

9. Henderson NC, et al. Targeting of $\alpha \mathrm{v}$ integrin identifies a core molecular pathway that regulates fibrosis in several organs. Nat Med. 2013;19(12):1617-1624.

10. Shi M, et al. Latent TGF- $\beta$ structure and activation. Nature. 2011;474(7351):343-349.

11. Munger JS, et al. The integrin $\alpha v \beta 6$ binds and activates latent TGF beta 1: a mechanism for regulating pulmonary inflammation and fibrosis. Cell. 1999;96(3):319-328.

12. $\mathrm{Mu} \mathrm{D}$, et al. The integrin $\alpha \mathrm{v} \beta 8$ mediates epithelial homeostasis through MT1-MMP-dependent activation of TGF- $\beta 1 . J$ Cell Biol. 2002;157(3):493-507.

13. Wipff PJ, Rifkin DB, Meister JJ, Hinz B. Myofibroblast contraction activates latent TGF- $\beta 1$ from the extracellular matrix. $J$ Cell Biol. 2007;179(6):1311-1323.

14. Murray IR, et al. av Integrins on mesenchymal cells regulate skeletal and cardiac muscle fibrosis. Nat Commun. $2017 ; 8(1): 1118$.

15. Bradley JE, Ramirez G, Hagood JS. Roles and regulation of Thy-1, a context-dependent modulator of cell phenotype. Biofactors. 2009;35(3):258-265.

16. Barker TH, Hagood JS. Getting a grip on Thy-1 signaling. Biochim Biophys Acta. 2009;1793(5):921-923.

17. Rege TA, Hagood JS. Thy-1 as a regulator of cell-cell and cell-matrix interactions in axon regeneration, apoptosis, adhesion, migration, cancer, and fibrosis. FASEB J. 2006;20(8):1045-1054.

18. Liu X, et al. Thy-1 interaction with Fas in lipid rafts regulates fibroblast apoptosis and lung injury resolution. Lab Invest. 2017(3):256-67.

19. Nicola T, et al. Loss of Thy-1 inhibits alveolar development in the newborn mouse lung. Am J Physiol Lung Cell Mol Physiol. 2009;296(5):L738-L750

20. Hagood JS, et al. Loss of fibroblast Thy-1 expression correlates with lung fibrogenesis. Am J Pathol. 2005;167(2):365-379.

21. Sueblinvong V, et al. Aging promotes pro-fibrotic matrix production and increases fibrocyte recruitment during acute lung injury. Adv Biosci Biotechnol. 2014;5(1):19-30.

22. Zhou Y, Hagood JS, Murphy-U1lrich JE. Thy-1 expression regulates the ability of rat lung fibroblasts to activate transforming growth factor-beta in response to fibrogenic stimuli. Am J Pathol. 2004;165(2):659-669.

23. Hagood JS, Lasky JA, Nesbitt JE, Segarini P. Differential expression, surface binding, and response to connective tissue growth factor in lung fibroblast subpopulations. Chest. 2001;120(1 suppl):64S-66S

24. Hagood JS, et al. Differential expression of platelet-derived growth factor-alpha receptor by Thy-1(-) and Thy-1(+) lung fibroblasts. Am J Physiol. 1999;277(1):L218-L224.

25. Hagood JS, Mangalwadi A, Guo B, MacEwen MW, Salazar L, Fuller GM. Concordant and discordant interleukin-1-mediated signaling in lung fibroblast thy-1 subpopulations. Am J Respir Cell Mol Biol. 2002;26(6):702-708

26. Sanders YY, Kumbla P, Hagood JS. Enhanced myofibroblastic differentiation and survival in Thy-1(-) lung fibroblasts. $A m J$ Respir Cell Mol Biol. 2007;36(2):226-235.

27. Liu X, et al. Thy-1 interaction with Fas in lipid rafts regulates fibroblast apoptosis and lung injury resolution. Lab Invest. 2017;97(3):256-267.

28. Zhou Y, Hagood JS, Lu B, Merryman WD, Murphy-Ullrich JE. Thy-1-integrin avß5 interactions inhibit lung fibroblast contraction-induced latent transforming growth factor- $\beta 1$ activation and myofibroblast differentiation. J Biol Chem 2010;285(29):22382-22393.

29. Fiore VF, Strane PW, Bryksin AV, White ES, Hagood JS, Barker TH. Conformational coupling of integrin and Thy-1 regulates Fyn priming and fibroblast mechanotransduction. J Cell Biol. 2015;211(1):173-190.

30. Frippiat C, Chen QM, Zdanov S, Magalhaes JP, Remacle J, Toussaint O. Subcytotoxic H2O2 stress triggers a release of transforming growth factor- $\beta 1$, which induces biomarkers of cellular senescence of human diploid fibroblasts. J Biol Chem. 2001;276(4):2531-2537

31. Neveu WA, Mills ST, Staitieh BS, Sueblinvong V. TGF- $\beta 1$ epigenetically modifies Thy-1 expression in primary lung fibroblasts. Am J Physiol Cell Physiol. 2015;309(9):C616-C626.

32. Xing S, et al. HDAC is essential for epigenetic regulation of Thy-1 gene expression during LPS/TLR4-mediated proliferation of lung fibroblasts. Lab Invest. 2015;95(10):1105-1116.

33. Fiore VF, et al. $\alpha v \beta 3$ Integrin drives fibroblast contraction and strain stiffening of soft provisional matrix during progressive fibrosis. JCI Insight. 2018;3(20):97597.

34. Rapisarda V, et al. Integrin $\beta 3$ regulates cellular senescence by activating the TGF- $\beta$ pathway. Cell Rep. 2017; 18(10):2480-2493

35. Lee CG, et al. Early growth response gene 1-mediated apoptosis is essential for transforming growth factor $\beta 1$-induced pulmonary fibrosis. J Exp Med. 2004;200(3):377-389.

36. Hagood JS. Thy-1 as an integrator of diverse extracellular signals. Front Cell Dev Biol. 2019;7:26

37. Ikonomou L, Wagner DE, Turner L, Weiss DJ. Translating basic research into safe effective cell-based treatments for respiratory diseases. Ann Am Thorac Soc. 2019;16(6):657-668. 
38. Barlow JZ, Kelley KA, Bozdagi O, Huntley GW. Testing the role of the cell-surface molecule Thy-1 in regeneration and plasticity of connectivity in the CNS. Neuroscience. 2002;111(4):837-852.

39. Ashcroft T, Simpson JM, Timbrell V. Simple method of estimating severity of pulmonary fibrosis on a numerical scale. J Clin Pathol. 1988;41(4):467-470. 